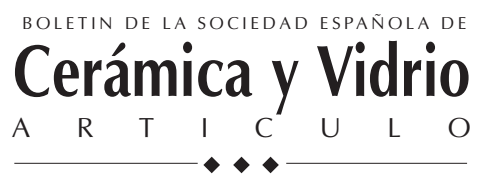

\title{
Conformado de materiales cerámicos por electroforesis en medios acuosos
}

\author{
B. FERRARI Y R. MORENO \\ Instituto de Cerámica y Vidrio, C.S.I.C. Ctra Valencia Km. 24,300; 28500 Arganda del Rey, Madrid
}

\begin{abstract}
La deposición electroforética es un proceso de conformado particularmente sencillo en el que, al hacer pasar una corriente a través de una suspensión coloidal o una disolución, se obtienen recubrimientos sobre materiales conductores. La mayor parte de los materiales orgánicos y minerales pueden ser procesados por electrodeposición, siendo la mayor dificultad la selección del vehículo líquido y el ajuste de las condiciones experimentales al sistema seleccionado. En el conformado por vía líquida el éxito del proceso radica en la preparación de suspensiones estables de determinadas características eléctricas. En este sentido el uso de vehículos orgánicos presenta grandes ventajas, ya que evitan en gran medida los procesos electrolíticos que tienen lugar en el entorno del electrodo, y que por tanto afectan a la homogeneidad y posible contaminación del recubrimiento. No obstante, el uso de orgánicos supone el encarecimiento de un proceso que se caracteriza por sus ventajas económicas y ecológicas. Por ello, los procesos de electroforesis a partir de medios acuosos, están recibiendo una creciente atención dadas las enormes posibilidades para su implantación en ciclos de producción de una amplia gama de materiales, como de hecho ocurre en la obtención de recubrimientos de arcillas y esmaltes. En este trabajo se lleva a cabo una revisión de la fisicoquímica del proceso así como de sus posibilidades de aplicación en la fabricación de productos cerámicos y vítreos en medio acuoso.
\end{abstract}

Palabras clave: Deposición, Electroforesis, Suspensiones, Recubrimientos.

\section{Electrophoretic forming of ceramics in aqueous media}

Electrophoretic deposition is a particularly easy forming technique in which coatings onto conducting electrodes are obtained after applying a dc electric field to a colloidal suspension or solution. Most organic materials and minerals can be deposited, being the biggest difficulty the selection of the organic vehicle and the adjustment of the experimental conditions. In wet forming processes, the success is determined by the preparation of stable suspensions with the required electrical properties. In this sense, the use of organics presents important advantages, since avoid electrolityc processes in the vicinity of electrodes, which highly affect the homogeneity and contamination of the coating. However, the use of organics increases the costs of a process characterized for its economical and environmental advantages. This is the reason why aqueous electrophoresis is receiving an increasing attention due to the great possibilities to be considered for production cycles for a broad variety of materials, as already occurs in the case of clays and enamels. In this work, a review of the physical chemistry of the process is made, and possible applications in ceramics and glasses manufacturing are evaluated.

Key words: Deposition, Electrophoresis, Suspensions, Coatings

\section{INTRODUCCIÓN}

El conformado por deposición electroforética, o electrodeposición (EPD), se enmarca dentro de los métodos de conformado por vía húmeda, en los cuales la preforma cerámica en verde se obtiene a partir de una suspensión de polvos, más o menos concentrada. En esta técnica en concreto, se preparan suspensiones coloidales de bajo contenido en sólidos (entre un $5 \%$ y un $20 \%$ en peso aunque, excepcionalmente, se ha descrito el uso de concentraciones superiores, hasta el 60\%), en comparación con otros tipos de procesamiento por vía húmeda, como el colaje en molde poroso, con o sin presión, o el colaje en cinta, en los que se alcanzan concentraciones en sólidos comprendidas entre el 65 y el $80 \%$ en peso.

La electroforesis es un fenómeno electrocinético asociado al movimiento de partículas que tiene lugar al aplicar un campo eléctrico sobre una suspensión coloidal estable. En el campo del procesamiento cerámico, se define la EPD como el proceso por el que se produce la migración de partículas suspendidas en un vehículo líquido, por efecto electroforético, y su posterior deposición sobre un electrodo. De esta forma se obtiene una densa capa de material cerámico sobre un sustrato conductor.

Este método de conformado viene siendo objeto de estudio desde la década de los 40 [1-3], siendo ya conocido desde tiempo atrás en pulvimetalúrgia. El interés que ha suscitado la EPD en los últimos años se debe fundamentalmente a que es una técnica versátil, económica y reproducible, en base a las innumerables posibilidades de diseño y a la simplicidad del equipo necesario.

El objetivo de este trabajo es introducir y discutir los fenómenos de electroforesis y deposición, y analizar los factores involucrados en la cinética del proceso. Así mismo, se hace una breve revisión de las aplicaciones actuales de la técnica en el 
conformado de piezas y recubrimientos, y se evalúan sus crecientes posibilidades en el diseño de materiales compuestos.

\section{DISEÑO Y EQUIPAMIENTO NECESARIO}

El equipamiento básico necesario no requiere fuertes inversiones, ya que consta de una sencilla célula electroforética a la que se acoplan varios equipos auxiliares, que se utilizan en el control del proceso. La figura 1 muestra un esquema de la célula y el equipamiento básico utilizado a escala laboratorio.

La célula electroforética consiste en un recipiente en el que se introducen la suspensión y los electrodos. Generalmente se utilizan dos electrodos, un contraelectrodo y un electrodo de trabajo o substrato sobre el cual se deposita el material. En algunas ocasiones, se utilizan electrodos de referencia para mantener el control de la propiedades eléctricas de la suspensión y del entorno del electrodo de trabajo. Los electrodos suelen estar unidos a soportes no conductores de forma que se mantenga el planoparalelismo y la distancia entre ellos, es decir, para mantener constante la geometría del sistema. De esta forma se asegura una intensidad de campo eléctrico constante con una distribución homogénea, en el volumen de suspensión que se encuentra entre ambos electrodos.

En cuanto a los equipos auxiliares, además de la fuente de potencia que mantiene las condiciones eléctricas del proceso, se utilizan agitadores, termostatos y potenciostatos. Los agitadores se utilizan para establecer un régimen estacionario en la suspensión y evitar la posible sedimentación de partículas por acción de la gravedad. En ocasiones, como veremos más adelante, las condiciones de temperatura son críticas en la viabilidad del proceso y en la obtención de materiales homogéneos, por lo que se utilizan termostatos para mantener la temperatura constante a lo largo del ensayo. Otras veces, sobre todo en ensayos de larga duración, se utilizan potenciostatos auxiliares para controlar la evolución de las propiedades eléctricas de la suspensión y de la interfaz electrodo/suspensión.

En comparación con las tecnologías láser, plasma, vacío, etc. que utilizan otras técnicas de obtención de recubrimientos, el equipo utilizado en la EPD resulta menos complejo y más económico. Por otro lado, frente a métodos más tradicionales de obtención de piezas cerámicas, como el moldeo o el colaje en molde de escayola [4-6], compite en costes, principalmente debido a que la duración del proceso es menor, ya que la cinética de la EPD es mucho más rápida que la de cualquiera de los otros tipos de procesamiento mencionados.

Por otra parte, la técnica de EPD resulta extraordinariamente versátil, ya que ofrece la posibilidad de obtener desde recubrimientos de decenas de micras, con diversidad de formas, hasta piezas de varios milímetros de espesor, tanto planas como huecas y de geometrías complejas. Esto se consigue simplemente utilizando el molde, substrato o electrodo adecuado, con la forma del material que se desea obtener. La única limitación es el diseño de una geometría adecuada para mantener un campo eléctrico homogéneo y constante a lo largo del proceso.

En la figura 2 se muestran varios diseños de electrodos y células de electrodeposición a escala de laboratorio, reportados en la bibliografía, tanto en el campo de la cerámica tradicional para la obtención de crisoles [7] (fig. 2a) y la aplicación de esmaltes [8] (fig 2b), como en cerámica avanzada para la obtención de recubrimientos de placas [9] (fig. 2c), hilos o tubos [10] (fig. 2d) y materiales no conductores o en infiltraciones [11] (fig. 2e). Esta figura ilustra las enormes posibilidades de aplicación de esta técnica para la manufactura de una

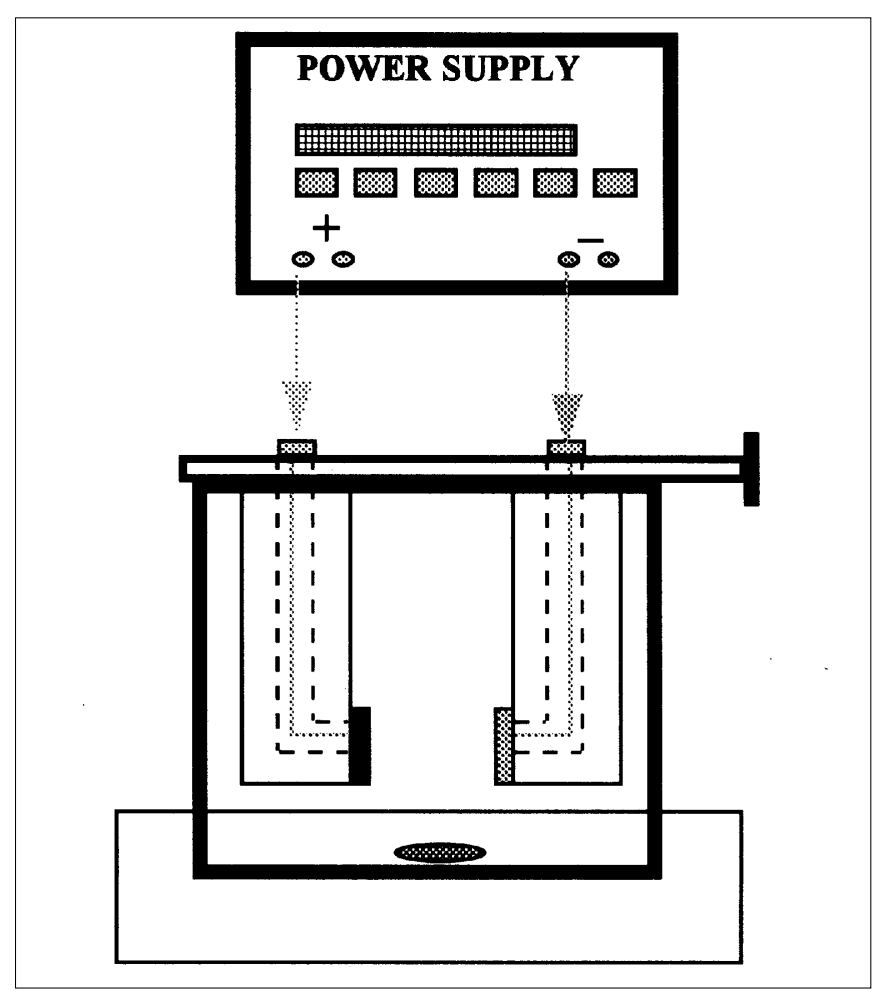

Fig. 1. Esquema del dispositivo de EPD.

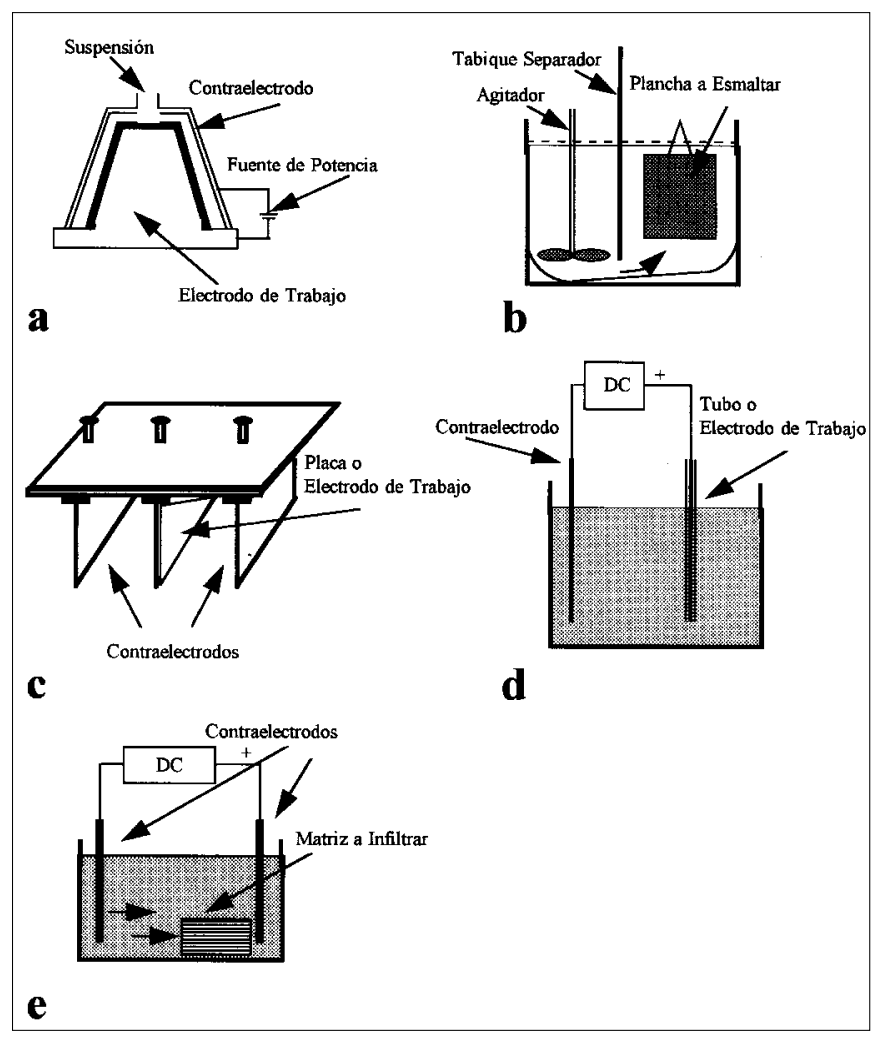

Fig. 2. Diseños de electrodos y células de electrodeposición para la obtención de crisoles (a), esmaltes (b), recubrimientos de placas (c), hilos o tubos (d) e infiltraciones sobre matriz porosa (e). 


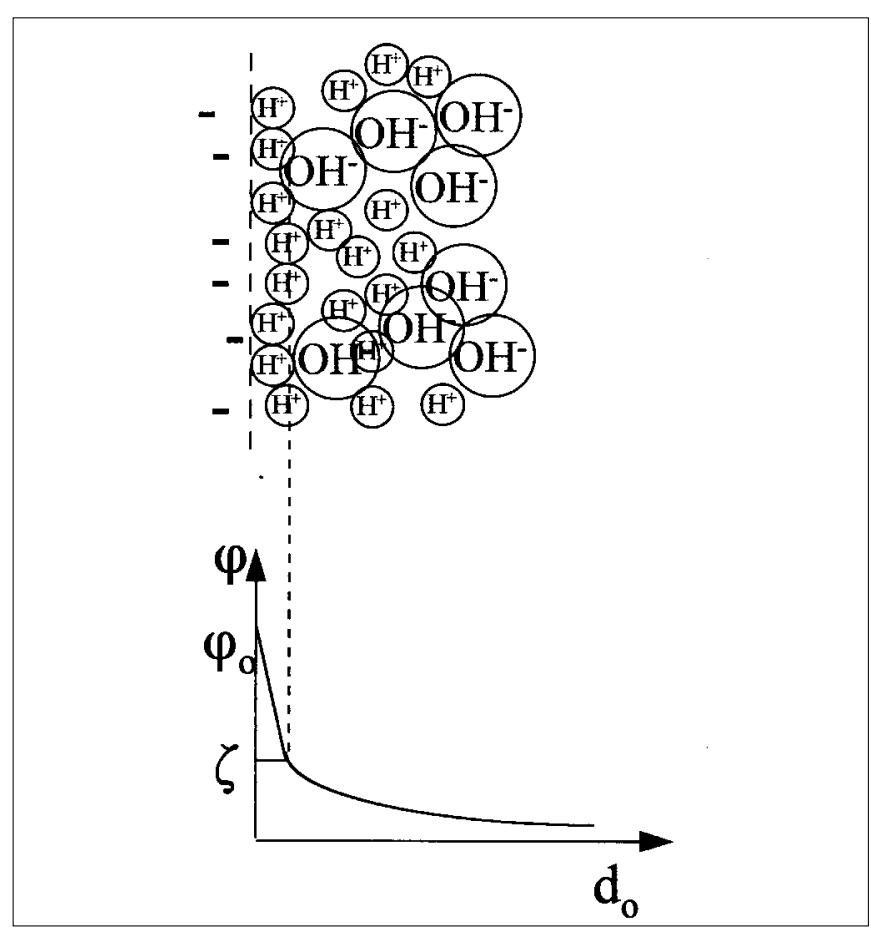

Fig. 3. Esquema de la doble capa eléctrica y la variación del potencial.

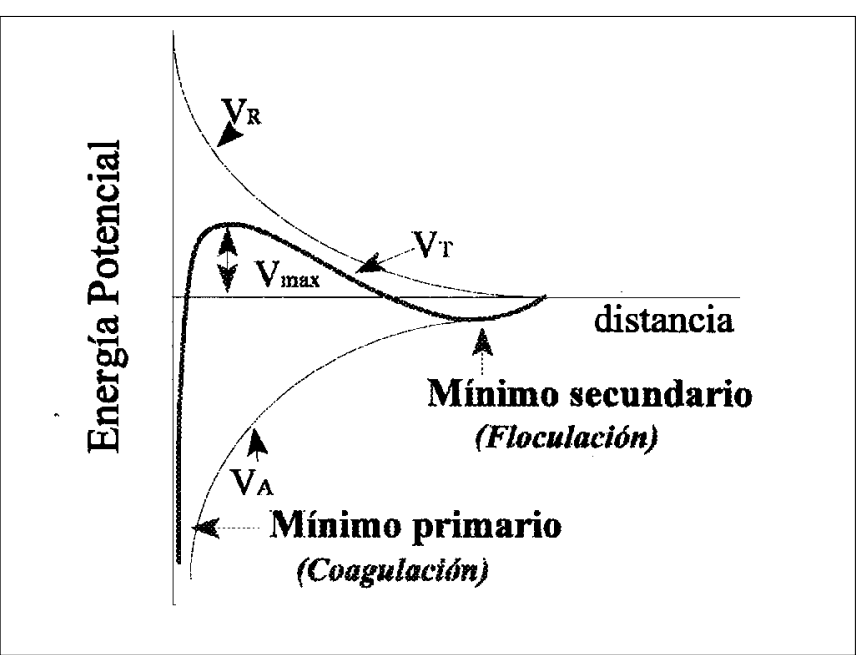

Fig. 4. Potencial de interacción entre partículas en función de la distancia entre ellas.

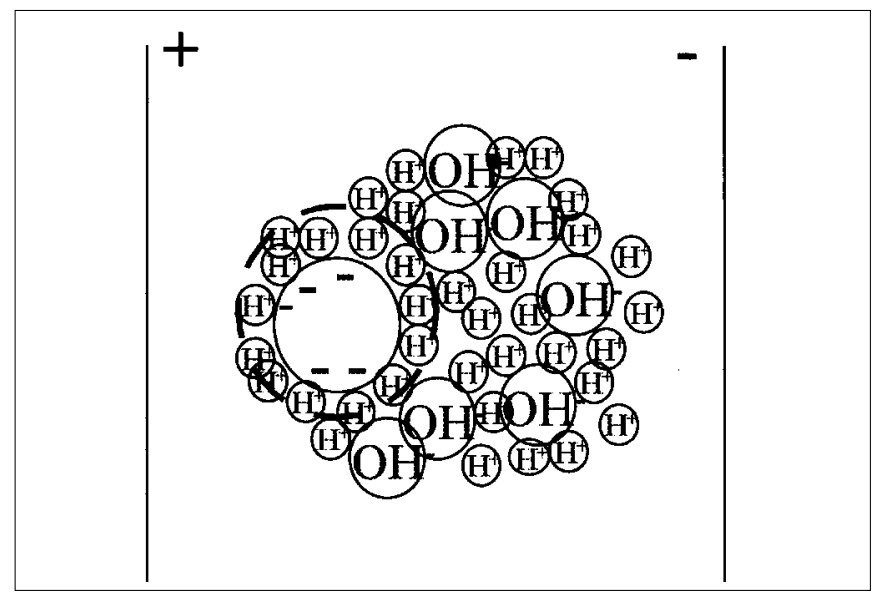

Fig. 5. Esquema de la distorsión de la doble capa durante la electroforesis de las partículas. gran diversidad de materiales, sean productos de cerámica tradicional o avanzada y, dentro de ésta, materiales compuestos, bien como substratos autosoportados o como recubrimientos.

A continuación se describirán brevemente los dos procesos que intervienen en la EPD, así como el desarrollo de la ecuación que determina la cinética del proceso.

\section{ELECTROFORESIS}

La EPD se basa en el movimiento, en el seno de un líquido, de partículas cargadas, originado al aplicar un campo eléctrico sobre una suspensión coloidal estable. Este fenómeno electrocinético recibe el nombre de electroforesis, y está directamente relacionado con las propiedades electroquímicas superficiales que adquieren las partículas cuando se hallan inmersas en un medio líquido.

En general, las partículas suspendidas en un líquido polar se cargan superficialmente. Las reacciones superficiales, y por tanto, el signo de la carga superficial de un polvo cerámico en suspensión acuosa dependen del pH del medio [12]. Alrededor de la partícula cargada se crea una doble capa iónica según establece la teoría de Stern [13]. Esta teoría propone que la partícula cargada superficialmente genera un potencial superficial $\Psi_{s}$, debido a lo cual atrae iones de signo contrario o contraiones. Los contraiones rodean la partícula formando una monocapa fuertemente adherida a la superficie de la misma, que recibe el nombre de capa rígida. Alrededor de esta monocapa, se crea una nube de iones de ambos signos que forman la capa difusa, en la que la concentración de contraiones va decreciendo al ir aumentando la distancia a la superficie de la partícula. De esta forma se mantiene la neutralidad electroquímica del sistema, ya que el potencial superficial desarrollado por la partícula cerámica en suspensión se compensa a medida que nos alejamos de su superficie por el efecto de la doble capa, tal y como muestra la figura 3.

La estabilidad electroquímica de las partículas viene determinada por la interacción partícula-líquido (doble capa) y partícula-partícula (interacción entre las dobles capas desarrolladas por las partículas) según la teoría de DLVO [12,13]. La figura 4 representa la variación de la energía potencial de interacción $\left(\mathrm{V}_{\mathrm{T}}\right)$ entre partículas en función de la distancia entre ellas, considerando solo la actuación de fuerzas de atracción $\left(V_{A}\right)$ y de repulsión $\left(V_{R}\right)$ electrostáticas. En la figura se observa que la curva energía potencial neta tiene dos mínimos que reflejan comportamientos típicos de las suspensiones: coagulación y floculación.

A distancias cortas predominan las fuerzas de atracción entre partículas, dando lugar a un mínimo primario, en el que las partículas cargadas entran en contacto unas con otras de forma irreversible, lo que produce la coagulación de la suspensión. A medida que la distancia entre partículas aumenta, predominan las fuerzas de repulsión. En este punto la gráfica presenta un pico o barrera de energía, en la que la repulsión entre partículas es máxima. A mayor distancia las fuerzas de atracción dominan de nuevo sobre las de repulsión, pero aunque las partículas se atraen mutuamente no entran en contacto directo, quedando una película de líquido entre ellas como resultado de una unión débil y reversible. Este último efecto esta representado en la curva de energía total como un mínimo secundario, no siempre presente, en el que tiene lugar el fenómeno de floculación.

La máxima estabilidad de la suspensión viene determinada por el punto de máxima repulsión entre partículas, directa- 
mente relacionado con la formación de sus dobles capas. De forma experimental se puede evaluar a través del potencial zeta o potencial electrocinético, $\zeta$, que es el valor del potencial medido en el plano de cizalla entre la capa rígida y la capa difusa de la doble capa (fig. 3).

La relación entre la estabilidad electroquímica de la suspensión y el movimiento de las partículas viene dado por la relación entre el potencial zeta y la movilidad electroforética $u_{e}$ de las mismas, según la ecuación de Henry:

$$
u_{e}=\frac{\zeta \varepsilon}{1,5 \eta} f\left(\chi_{r_{p}}\right)
$$

donde $\varepsilon$ es la constante dieléctrica del medio y $\eta$ su viscosidad, y $f\left(\chi_{r}\right)$ es un parámetro que depende de la forma de la doble capa, y que toma el valor 1 en caso de considerar partículas perfectamente esféricas [14].

En consecuencia, al hacer pasar una densidad de corriente determinada a través de una suspensión estable, se crea un campo eléctrico tangencial a la superficie cargada de las partículas, ejerciendo una fuerza en las dos porciones de la doble capa. La superficie cargada y la capa de iones unidos rígidamente a ella, tienden a moverse en función del signo de su potencial (potencial zeta), según la dirección del campo creado, mientras que los iones que están fuera de la superficie de cizalla (capa difusa) tienden a orientarse en sentido opuesto, tal y como se representa en la figura 5, para una partícula cargada negativamente en superficie.

En conclusión, para asegurar un flujo de partículas uniforme y potencialmente depositables sobre un substrato se debe tener en cuenta : 1) que la suspensión sea suficientemente estable, dotando a las partículas de una elevada movilidad electroforética, y 2) que el campo eléctrico creado sobre la suspensión sea homogéneo, en función de la geometría del sistema, y lo suficientemente elevado para hacer que las partículas se muevan en la dirección deseada, aplicando la densidad de corriente adecuada.

\section{DEPOSICIÓN}

El segundo proceso que tiene lugar durante el conformado es la deposición de las partículas sobre el electrodo por acción de un campo eléctrico (figura 6). Para que las partículas se depositen formando una capa compacta con cohesión interna, la suspensión debe hacerse inestable en el entorno del electrodo. La pérdida de estabilidad se traduce en la disminución del espesor de la doble capa de las partículas que llegan al entorno del electrodo, permitiendo la suficiente aproximación entre ellas como para que predominen las fuerzas de atracción de Van der Waals, forzando así la coagulación del sistema.

En la formación del depósito sobre el electrodo intervienen varios fenómenos paralelos. En relación a la suspensión propiamente dicha, la distorsión que sufre la doble capa al desplazarse la partícula en el líquido, el aumento del contenido en sólidos por acumulación de partículas cerca del electrodo y la reformación de las moléculas del electrolito y de los aditivos, son los principales responsables de la pérdida de estabilidad de la suspensión y, por tanto, de la formación del depósito [15].

No obstante, según la teoría DLVO, la coagulación de las

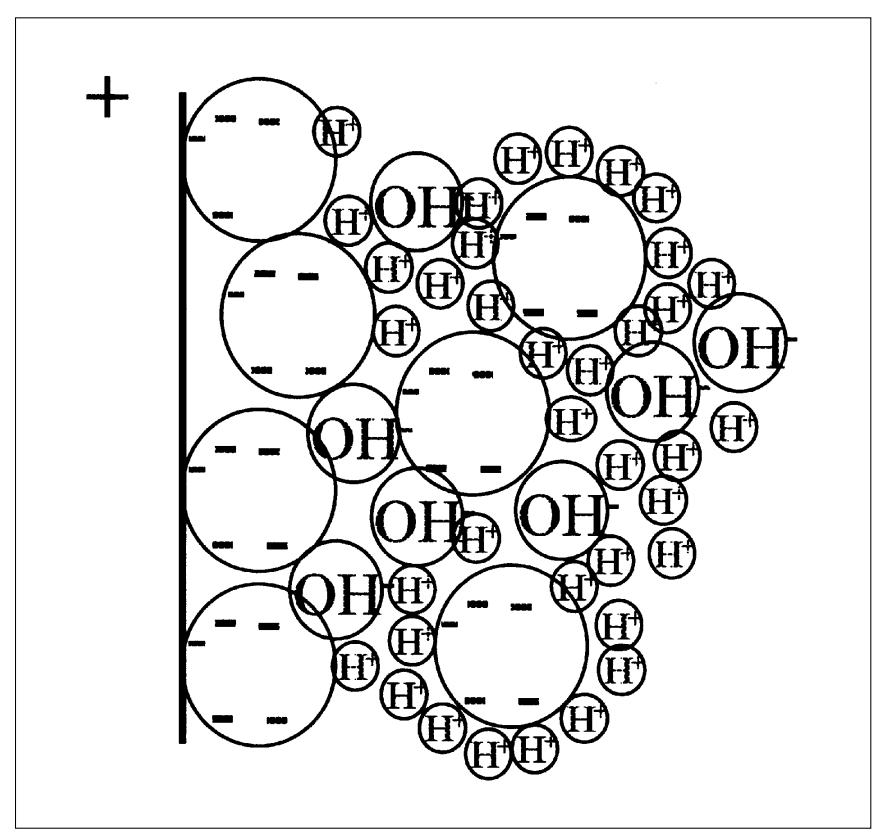

Fig. 6. Esquema de la deposición de las partículas en el electrodo.

partículas viene determinada por la fuerza de interacción necesaria para superar la barrera de energía de repulsión (fig 4) [13]. En la figura 7 se muestra un esquema de balance de fuerzas que actúan sobre las partículas para forzar su coagulación en el entorno del electrodo [16]. A la fuerza de interacción electrostática $\left(\mathrm{F}_{\mathrm{T}}\right)$ que actúa sobre las partículas se suma la fuerza electroforética $\left(\mathrm{F}_{\mathrm{E}}\right)$ derivada del campo eléctrico aplicado a la suspensión.

En consecuencia, en un sentido estricto, el mecanismo de deposición propiamente dicho se basa en la pérdida de estabilidad de la suspensión y en la formación de un depósito con cohesión interna. Es en la relajación de la doble capa donde intervienen procesos de transferencia de carga típicos de las reacciones de interacción eléctrica de la materia. La elección de un determinado sistema, como el tipo de electrodo utilizado (metálico, semiconductor, etc.) o el medio en el que se suspenden las partículas (orgánico o acuoso), puede dar lugar a varios mecanismos que favorezcan la descarga de la doble capa.

\section{EVOLUCIÓN DEL DEPÓSITO}

\subsection{Cantidad de material depositado}

En función del elevado número de procesos paralelos que pueden llegar a tener lugar en el electrodo de trabajo o substrato a lo largo del proceso, puede considerarse la electrodeposición como un proceso no faradáico, ya que a la transferencia de carga que tiene lugar en ambos electrodos, esta asociada otra serie de fenómenos como el transporte de masa y deposición, que provocan cambios (de potencial, de concentración de la suspensión, etc.) en la interfaz electrodo/suspensión. Por otra parte, los fenómenos de electrodo que tienen lugar al margen de la deposición electroforética propiamente dicha, pueden hacer disminuir en mayor o menor medida el rendimiento del proceso, ya que parte de la corriente suministrada se pierde en promoverlos. 


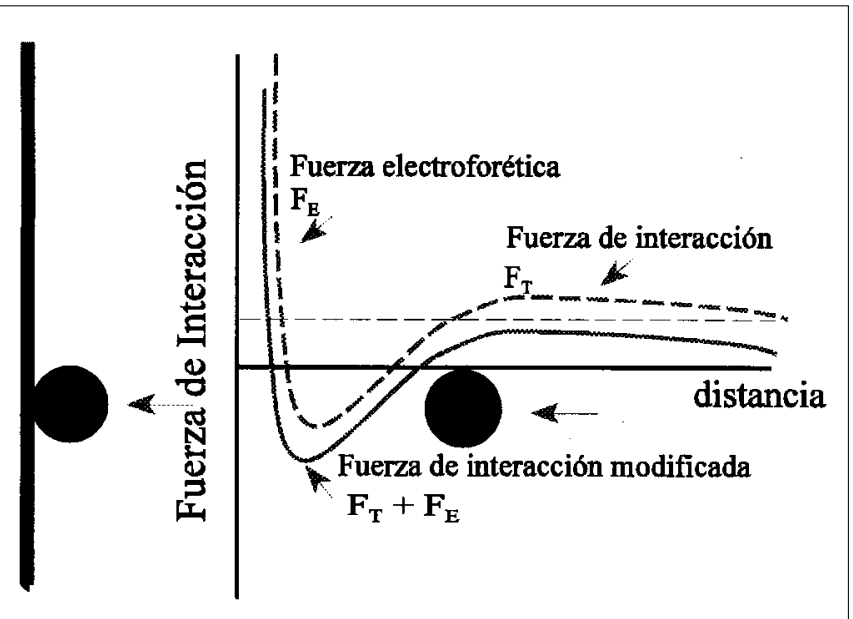

Fig. 7. Fuerzas de interacción en el entorno del electrodo. pensión y $n_{i}$ el número de partículas por unidad de volumen. De esta manera la cantidad de masa depositada viene dada por la expresión:

$$
m=\frac{q_{i} C_{i}}{n_{i} z_{i} e}
$$

La cantidad de carga puede definirse como la intensidad de corriente, $I(\mathrm{~A})$, aplicada sobre las i partículas en suspensión en un tiempo $t(\mathrm{~s})$, de tal forma que:

$$
m=\frac{C}{n_{i} z_{i} e} I t
$$

ec. 5.5

La intensidad del campo eléctrico creado en la suspensión es inversamente proporcional a la intensidad de corriente aplicada, tal que:

$$
E=\frac{I}{\sigma A}
$$

ec. 5.6

Aún no siendo un proceso faradáico, en un caso ideal en el que suponemos que la densidad de corriente suministrada a la suspensión es la misma durante todo el proceso y no hay pérdida de concentración de polvo, se puede suponer que la masa depositada es directamente proporcional a la cantidad de carga que se hace pasar a través de la suspensión [10], de forma que:

$$
m \approx Q
$$

donde $m$ es la cantidad de masa depositada (g), y $Q$ la cantidad de carga que transportan las i partículas en suspensión (C).

La cantidad de masa depositada por cada portador de carga i, por unidad de área del electrodo es:

$$
\frac{m}{M}=\frac{q_{i}}{z_{i} N e}
$$

ec. 5.2

donde $\mathrm{m}$ es la cantidad de masa depositada, $\mathrm{M}$ es la masa de las i partículas del número de Avogrado (N), $\mathrm{q}_{\mathrm{i}}$ la carga eléctrica transportada por las i partículas en suspensión, $z_{i}$ es la carga de la partícula, y e la carga electrónica.

Dado que $\mathrm{M} / \mathrm{N}$ es la masa de cada partícula, se puede redefinir como:

$$
\frac{M}{N}=\frac{C_{i}}{n_{i}}
$$

donde $C_{i}$ es la concentración volumétrica $\left(\mathrm{g} \mathrm{cm}^{-3}\right)$ de la sus-

$$
u_{e}=\frac{\sigma}{n_{i} z_{i} e}
$$

donde $E$ es la intensidad de campo eléctrico $\left(\mathrm{V} \mathrm{cm}^{-1}\right)$, $\sigma$ es la conductividad eléctrica de la suspensión $\left(\mathrm{S} \mathrm{cm}^{-1}\right), \mathrm{y} A$ es el área del electrodo $\left(\mathrm{cm}^{2}\right)$.

La cantidad de masa depositada viene dada por la expresión:

$$
m=\frac{C}{n_{i} z_{i} e} \sigma A E t
$$

En electroquímica de sistemas coloidales [17] se define la movilidad electroforética, $u_{e}\left(\mathrm{~cm}^{2} \mathrm{~s}^{-1} \mathrm{~V}^{-1}\right)$, como:

por lo que nos queda:

$$
m=C u_{e} A E t
$$

expresión similar a la propuesta ya en el año 1940 por Hamaker y col.[1], en la que se asigna al parámetro $u_{e}$ un significado estadístico, como el número de partículas que logran alcanzar el electrodo.

Tal y como se propuso anteriormente, suponiendo que las partículas en suspensión tengan forma esférica, la movilidad electroforética se define en función del potencial zeta según la 
expresión 3.1, de tal forma que:

$$
m=\frac{C \varepsilon \zeta}{1,5 \eta} A E t
$$

Así pues, puede concluirse que la cantidad de masa depositada es función de la propiedades eléctricas del líquido que actúa como vehículo de suspensión (constante dieléctrica y viscosidad), de las propiedades de la suspensión coloidal (concentración de partículas), de las propiedades superficiales de las partículas (potencial zeta) y de la geometría y los parámetros eléctricos impuestos sobre el sistema (área, intensidad de campo eléctrico y tiempo).

\subsection{Cinética}

En el epígrafe anterior se ha visto que el número de parámetros que intervienen en el proceso de electrodeposición es elevado. Se ha comprobado experimentalmente $[9,10,18-21]$ que en efecto existe una relación lineal entre la masa depositada y el tiempo que se mantiene el campo eléctrico aplicado sobre la suspensión, relación que ya establece la ecuación 5.9. No obstante, algunos autores han encontrado en la práctica desviaciones de este comportamiento lineal teórico.

Tanto las condiciones eléctricas impuestas sobre el sistema como las características de la suspensión, han sido objeto de discusión en la bibliografía, teniendo siempre como objetivo precisamente conservar la linealidad en la cinética del proceso. Pero si bien los resultados experimentales son abundantes, hasta hace poco tiempo no se ha realizado un estudio teórico sobre la cinética del proceso. Sarkar y col. en 1996 [16] han hecho un balance de los estudios realizados sobre el tema hasta el momento, relacionando algunos de los resultados experimentales obtenidos en su propia investigación, con las condiciones impuestas al sistema.

Como resultado, definen fundamentalmente dos causas por las cuales la cinética se desvía del comportamiento lineal y el crecimiento del depósito se vuelve asintótico con el tiempo: 1) la disminución de la concentración en sólidos de la suspensión durante el ensayo y, 2) la caída de la densidad de corriente que la atraviesa, cuando el ensayo se realiza aplicando una diferencia de potencial constante entre los electrodos. Ambos problemas derivan del crecimiento del depósito sobre el electrodo a medida que el ensayo progresa.

En la figura 8 se representa el tipo de comportamiento esperado a medida que se van considerando los posibles defectos del ensayo. La relación lineal (fig. 8 curva 1) está sujeta a las condiciones ideales descritas en el epígrafe anterior. Es decir, la cantidad de masa depositada sobre el electrodo es directamente proporcional al tiempo, si se aplica sobre la suspensión una densidad de corriente constante, y se asegura que la pérdida de polvo en suspensión, debido al constante crecimiento del depósito, es despreciable.

La variación de la concentración de polvo en suspensión durante el ensayo $(\mathrm{C}=\mathrm{C}(\mathrm{t}))$ repercute sobre las condiciones electroquímicas de la suspensión preparada para llevar a cabo el ensayo, y hace disminuir el rendimiento del proceso, de forma que el crecimiento del depósito se hace asintótico con el tiempo (fig. 8 curva 2 ).

Este comportamiento se hace más pronunciado si, aún ase-

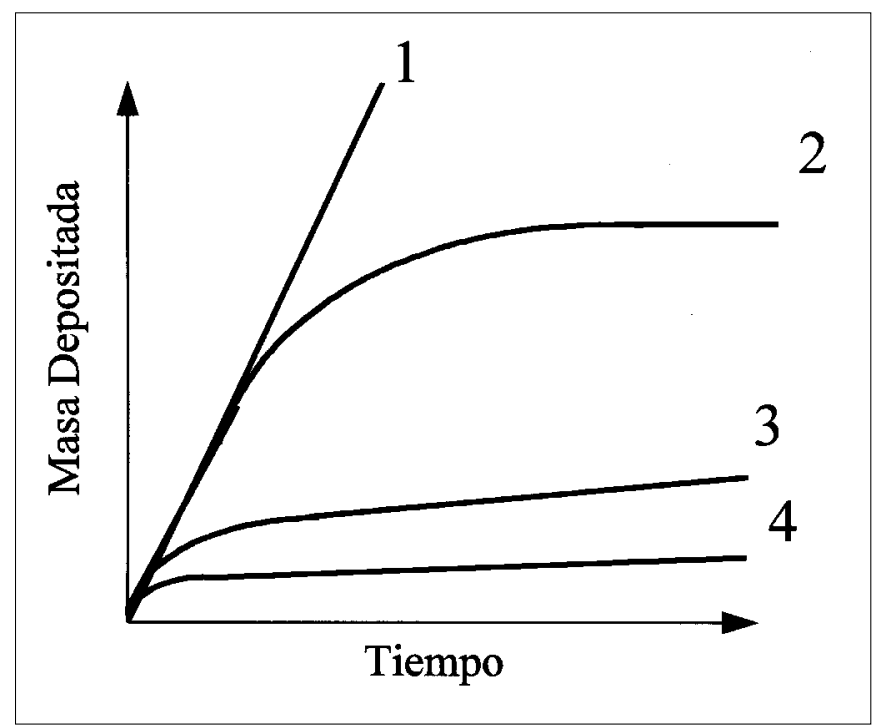

Fig. 8. Evolución de la masa depositada con el tiempo en condiciones de corriente y concentración constantes (1), corriente constante y concentración variable (2), voltaje y concentración constantes (3) y voltaje constante y concentración variable (4).

gurando una cantidad de polvo en suspensión suficiente para obviar el fenómeno descrito en el párrafo anterior, no aseguramos una intensidad de campo eléctrico homogénea y constante sobre la suspensión (fig. 8 curva 3). En este aspecto hemos de considerar que, en la mayoría de los trabajos consultados, los ensayos de EPD se realizan aplicando una diferencia de potencial constante entre los electrodos. En estas condiciones, el crecimiento de un depósito de considerables propiedades dieléctricas, como es el caso que nos ocupa, hará disminuir la densidad de corriente que se hace pasar a través de la suspensión, con lo que el campo eléctrico aplicado también disminuirá en intensidad, tendiendo a cero.

La caída de la intensidad de campo afecta tanto a la velocidad electroforética, $\mathrm{v}_{\mathrm{e}}\left(\mathrm{cm} \mathrm{s}^{-1}\right)$ de las partículas, que viene definida por la expresión:

$$
\mathrm{v}_{\mathrm{e}}=\mathrm{u}_{\mathrm{e}} \mathrm{E}
$$

como a la deposición de las partículas sobre el electrodo, como vimos anteriormente.

No obstante, hemos de tener en cuenta que la cinética de la EPD es distinta en cada sistema suspensión/substrato, por lo que, como ocurre en algunos trabajos reseñados en la bibliografía, es posible que aún sometiendo a la suspensión a un ensayo a voltaje constante, la cinética presente un comportamiento lineal a tiempos cortos. Nos encontraríamos dentro del primer tramo de la curva 3 representada en la figura 8 .

Por último, si tienen lugar ambos fenómenos simultáneamente, tendremos que la cinética del proceso abandonará en un periodo de tiempo aún más corto su comportamiento lineal inicial (fig. 8 curva 4 ). 

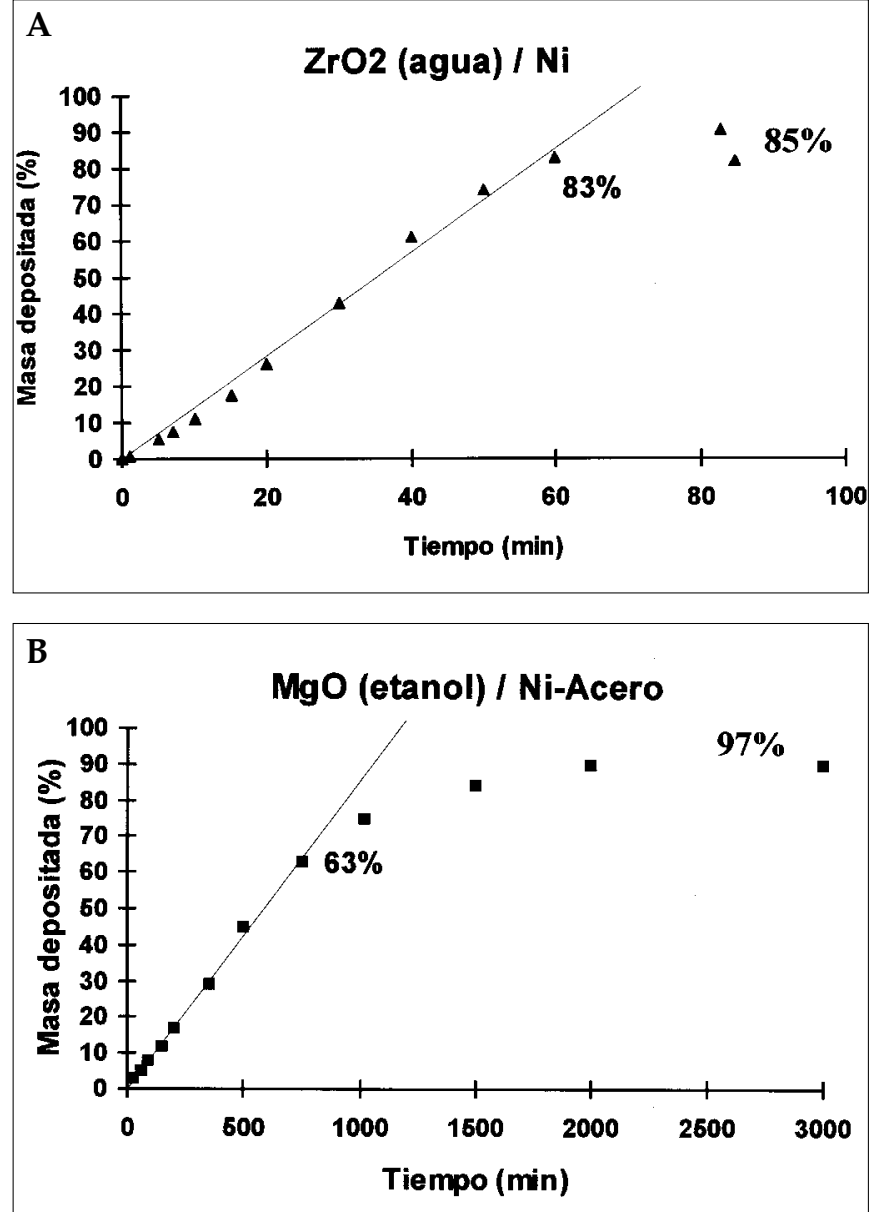

Fig. 9. Cinética de deposición de suspensiones acuosas de $\mathrm{ZrO}_{2}$ (a) y de suspensiones orgánicas de $\mathrm{MgO}$ (b) sobre electrodos de $\mathrm{Ni}$.

\section{CONFORMADO POR DEPOSICIÓN ELECTROFORÉTICA A PARTIR DE SUSPENSIONES ACUOSAS.}

\subsection{Ventajas e inconvenientes}

La utilización de $\mathrm{H}_{2} \mathrm{O}$ como vehículo líquido de la suspensión reporta una serie de ventajas relacionadas fundamentalmente con el abaratamiento de los costes y con los beneficios ecológicos del proceso de fabricación. Además del ahorro económico derivado del uso de agua destilada, cuyo precio es mínimo frente a las 1000 Ptas por litro de etanol (el etanol está gravado con impuesto especial de alcoholes), hay que tener en cuenta el ahorro de tiempo y espacio adicionales que reporta el proceso propiamente dicho. Por una parte, la cinética del proceso realizado en medio acuoso es mucho más rápida, ya que la constante dieléctrica de la mayoría de los disolventes orgánicos es menor que la del agua (ec. 5.10), y por otra parte, en relación al control del proceso, la conductividad térmica del agua es mayor que la de los disolventes orgánicos, lo que facilita el control de la temperatura, parámetro asociado directamente a la conductividad de la suspensión que limita el paso de corriente a través del sistema.

Sin embargo, el uso de $\mathrm{H}_{2} \mathrm{O}$ acarrea una serie de efectos derivados de fenómenos electroquímicos que tienen lugar en el entorno de los electrodos al hacer pasar la corriente eléctrica a través de la suspensión, y que hacen disminuir el rendimiento de la deposición. Los efectos de pérdida de rendimiento y aumento de la velocidad de deposición en ensayos realizados a partir de suspensiones acuosas u orgánicas, se hacen patentes al comparar las curvas a y b de la figura 9. La figura 9a representa la cantidad de $\mathrm{ZrO}_{2}$ depositada sobre un electrodo de $\mathrm{Ni}$ en relación a la cantidad de polvo suspendido en agua, respecto del tiempo que dura el ensayo trabajando en un rango de potenciales de 35 a $105 \mathrm{~V}$ [22]. La curva de la figura $9 \mathrm{~b}$ representa la misma evolución, trabajando en un rango de potenciales de 50 a $150 \mathrm{~V}$, sobre una suspensión en etanol de polvos de $\mathrm{MgO}$ depositados sobre Ni. Comparando ambas gráficas puede observarse cómo en agua sólo se llega a depositar aproximadamente un 85\% del polvo en suspensión, mientras que en etanol se alcanza a depositar el $97 \%$ del polvo suspendido [23]. No obstante, mientras que en agua ese $85 \%$ se alcanza en 80 minutos, en orgánicos el mismo porcentaje se alcanza en un ensayo de 1500 minutos, es decir utilizando un tiempo de deposición casi 20 veces mayor.

En cuanto a las reacciones que tienen lugar paralelamente a la deposición de las partículas en el electrodo, son: electrolisis y electroósmosis del agua, y reacciones galvanostáticas propias de la disolución del electrodo, en el caso de utilizar un electrodo metálico.

Según sea la polaridad de la célula electroforética pueden producirse las reacciones de electrolisis del agua:

$$
\begin{aligned}
& 2 \mathrm{H}_{2} \mathrm{O}+2 \mathrm{e}^{-} \rightleftharpoons \uparrow \mathrm{H}_{2}+2 \mathrm{OH}^{-} \\
& 4 \mathrm{OH}^{-}+2 \mathrm{H}_{2} \mathrm{O} \rightleftharpoons \mathrm{O}_{2}+4 \mathrm{e}^{-}
\end{aligned}
$$

provocando el desprendimiento de $\mathrm{H}_{2}$ en el cátodo y de $\mathrm{O}_{2}$ en el ánodo, burbujeo que puede llegar a dañar el depósito de forma irreversible haciendo que éste pierda su homogeneidad. Si bien este problema existe y puede comprobarse a simple vista durante el ensayo de EPD, resulta sorprendente comprobar la escasa repercusión que tiene sobre la densidad en verde de las muestras obtenidas bajo unas condiciones eléctricas controladas. En este sentido, se ha descrito en la bibliografía cómo a partir de un ensayo de EPD de $\mathrm{Al}_{2} \mathrm{O}_{3}$ sobre grafito [24], aplicando densidades de corriente desde 1,6 a $12,8 \mathrm{~mA} \mathrm{~cm}^{-2}$, se obtienen depósitos con densidades en verde dentro de un rango de $53-55 \%$. La figura 10 muestra la microestructura del depósito de $\mathrm{Al}_{2} \mathrm{O}_{3}$ obtenido en el ensayo realizado aplicando $12,8 \mathrm{~mA} \mathrm{~cm}^{-2}$ durante 10 minutos, en la que se puede comprobar la elevada homogeneidad de un depósito obtenido a partir de una suspensión acuosa de dicho polvo.

En cuanto a las reacciones de electrodo, en el caso de utilizar electrodos metálicos, hemos de tener en cuenta que debido a la elevada resistividad de la suspensiones utilizadas, en el entorno del electrodo se sobrepasa el potencial normal de cualquier metal. Cuando las partículas cargadas superficialmente migran y se depositan sobre el ánodo, las reacciones de oxidación del electrodo o substrato se generan prácticamente desde el primer segundo de ensayo. La formación de cationes metálicos que migran en sentido opuesto a las partículas, provocan la contaminación metálica de los depósitos formados. Dicha contaminación se hace patente en el deterioro del material en la intercara metal-cerámica, o incluso reaccionan con el material cerámico durante la sinterización, llegando en ocasiones a colorear el recubrimiento [22]. La micrografía de la figura 11 ilustra el deterioro de una pieza de $\mathrm{Al}_{2} \mathrm{O}_{3}$ obtenida por deposición sobre $\mathrm{Zn}$, en la que puede observarse cómo la porosidad generada por la volatilización de Zn en el proceso de sinterización va aumentando a medida que nos acercamos al borde de la muestra que estuvo en contacto con el electrodo [25]. 
Por último, ha de tenerse en cuenta el fenómeno de electroósmosis del $\mathrm{H}_{2} \mathrm{O}$, relacionado con las altas densidades en verde de las piezas obtenidas sobre todo en el campo de la cerámica tradicional. La electroósmosis es un fenómeno electrocinético por el que se produce el movimiento de la fase líquida (en este caso el $\mathrm{H}_{2} \mathrm{O}$ ) que impregna las partículas sólidas bajo el efecto de un campo eléctrico. La electroósmosis se produce en sentido inverso a la electroforesis, por lo que repercute en el secado gradual de las piezas a lo largo del ensayo de EPD. Si bien este proceso se plantea como una ventaja de la técnica en agua, ha de observarse con atención, ya que una duración excesiva del proceso puede provocar la formación de un gradiente de secado en la pieza que puede perjudicar el desmoldeo y provocar agrietamientos.

Si bien todos los fenómenos paralelos a la EPD propiamente dicha sobre los que se ha reflexionado hasta el momento parecen repercutir sobre el control del proceso, los datos experimentales muestran que en ocasiones no es así. En la figura 9 puede comprobarse cómo en medio acuoso (a), la cinética pierde su linealidad al depositarse un $83 \%$ (casi el máximo rendimiento que puede alcanzarse en estas condiciones) del polvo suspendido, es decir, el proceso no acusa el efecto producido por la pérdida de polvo o el deterioro de la suspensión a lo largo de prácticamente todo el tiempo de deposición [22]. Mientras que en el caso del etanol (b), la linealidad se pierde cuando se ha depositado tan solo un $63 \%$ del polvo suspendido [23]. En contrapartida, la lentitud del proceso hace que en medios orgánicos sea más fácil el control de depósitos de menor espesor y cantidad de masa depositada.

No obstante, se ha comprobado que en agua pueden llegar a controlarse también espesores de pocas decenas de micras. Un ejemplo de esto último, es la posibilidad de formación de estructuras laminadas $\mathrm{Al}_{2} \mathrm{O}_{3} / \mathrm{ZrO}_{2}$ como la que se muestra en la figura 12, en las que el espesor total de la estructura ya sinterizada es de $250 \mu \mathrm{m}$ y las distintas capas alcanzan espesores de 15 a $30 \mu \mathrm{m}[26]$.

De esta forma, no es extraño pensar que si bien el procesamiento por EPD en medio acuoso presenta tanto ventajas como inconvenientes, su importante connotación económica le ha llevado a experimentar un gran desarrollo, desde la década de los 80, en la industria de la cerámica tradicional (esmaltes, tazas, baldosas, sanitarios, etc.), donde las prestaciones que se piden a un material presentan límites de tolerancia más amplios, y por lo tanto, se requiere un control de la cinética menos preciso que en el campo de la cerámica avanzada. En este último campo, si bien la mayoría de los trabajos realizados se hacen a partir de suspensiones orgánicas, en los últimos años se han desarrollado incluso materiales de estructura compleja: materiales compuestos de matriz cerámica, estructuras con función gradiente, etc., a partir de sistemas acuosos, como veremos en el apartado 6.4.

6.2 Cerámica tradicional. Obtención de piezas por EPD a partir de barbotinas preparadas en medio acuoso

En la producción de piezas obtenidas a partir de materiales arcillosos de la industria cerámica se vienen utilizando métodos de conformado tradicionales, como prensado axial o isostático, extrusión, colaje en molde de yeso, etc., en función de los requerimientos de las piezas fabricadas. No obstante, métodos de procesamiento que parecían extremadamente costosos, han podido desarrollarse gracias al gran avance científico y tecnológico de las dos últimas décadas. Es el caso del cola-

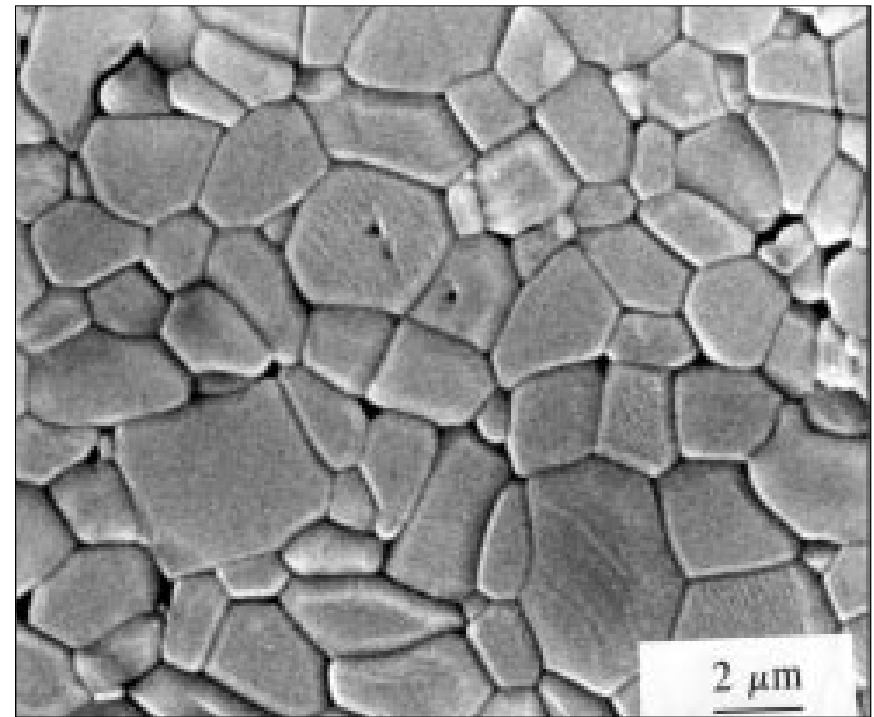

Fig. 10. Microestructura de un material sinterizado de $\mathrm{Al}_{2} \mathrm{O}_{3}$ obtenido por EPD sobre grafito.

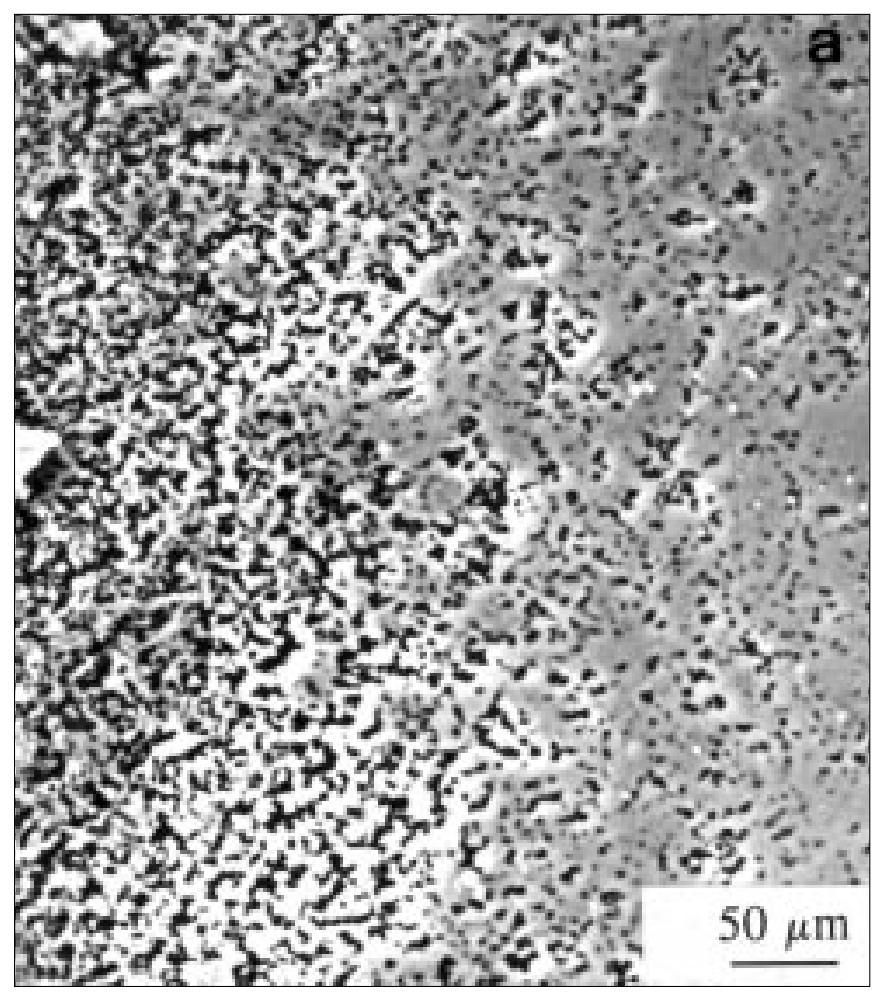

Fig. 11. Microestructura de la zona de la intercara de un material sinterizado de $\mathrm{Al}_{2} \mathrm{O}_{3}$ obtenido por EPD sobre un metal, en la que se observa la porosidad remanente consecuencia de la contaminación metálica del electrodo. 


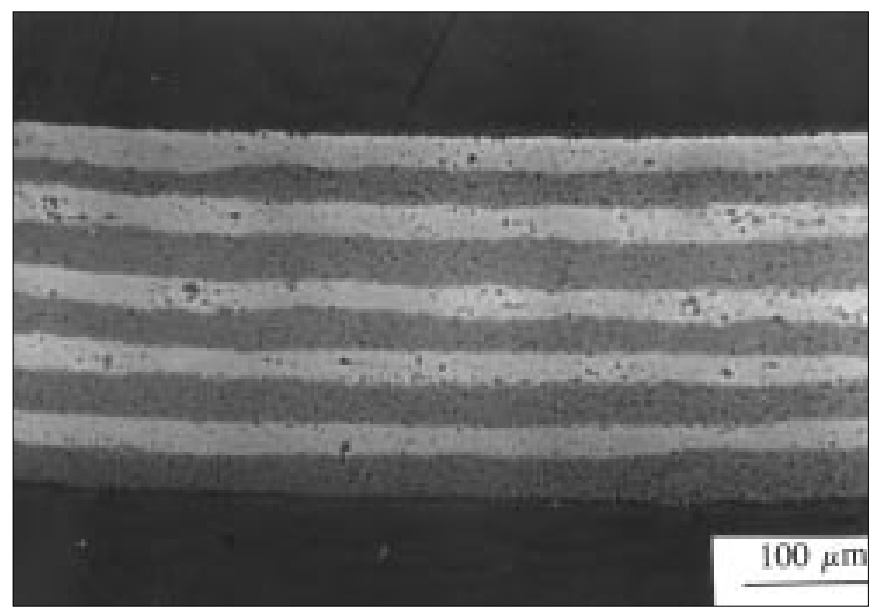

Fig. 12. Laminado $\mathrm{Al}_{2} \mathrm{O}_{3} / \mathrm{ZrO}_{2}$ obtenido por EPD en agua.

je con presión, el moldeo por inyección o el conformado por electroforesis con [27] y sin presión [4-7].

Como se ha explicado anteriormente, la EPD precisa de la preparación de una suspensión coloidal o barbotina en la que las partículas de polvo cerámico se encuentren perfectamente estabilizadas. Por lo tanto, los objetivos principales de la industria cerámica que desarrolla este tipo de procesamiento son la caracterización de suspensiones concentradas (estudios reológicos, electroquímicos, electrocinéticos, etc.) junto con la determinación de las condiciones adecuadas que permitan el control de procesamiento. En relación a los parámetros que determinan éstas, frente a técnicas tradicionalmente utilizadas como el colaje en molde de yeso y el prensado, o más novedosas, como el colaje con presión, la EPD muestra una serie de ventajas que hacen de ella una técnica más económica, versátil y controlable.

En primer lugar, teniendo en cuenta que la concentración en sólidos de las suspensiones preparadas en EPD es menor que en otro tipo de técnicas, también los rangos de los parámetros de la suspensión que determinan las condiciones óptimas del proceso son menos estrictos. Por ejemplo, la reología no es un parámetro tan crítico como lo es en las técnicas de filtración, por lo que se toleran rangos de viscosidades más amplios. Frente al colaje [4,5], el conformado por EPD no depende de la fluidez de la barbotina, ya que la fuerza que ejerce la presión capilar en colaje, es sustituida en EPD por la fuerza generada por un campo eléctrico aplicado sobre la suspensión desde el exterior. Por ello, si bien la viscosidad es importante de cara a asegurar la estabilidad de la suspensión, la capacidad de movimiento de las partículas suspendidas en el líquido y la fuerza con la que se mueven, es decir su velocidad electroforética, determinan con más exactitud la viabilidad del proceso.

Por otra parte, los moldes que se utilizan en EPD son más duraderos que los moldes de yeso, ya que son más resistentes a la abrasión y pueden ser secados a $100^{\circ} \mathrm{C}$ sin que pierdan sus propiedades fundamentales, como es la conductividad. Así pues, el proceso de secado de moldes ocupa menos tiempo y espacio en la cadena de producción, lo que significa un importante ahorro económico y energético. No obstante, hay que considerar los problemas que plantean los moldes debido a su carácter metálico al ser utilizados en un proceso electroquímico en presencia de agua. Para ello se han realizado estudios sobre el diseño de moldes [5,28,29], fabricándose, por ejemplo, moldes porosos tales que el tamaño de poro sea lo suficientemente grande para permitir el desalojo de las burbujas de $\mathrm{O}_{2}$ producidas por electrolisis del agua, pero suficientemente pequeños como para evitar un secado demasiado rápido de la pieza que perjudique el desmoldeo o provoque su agrietamiento.

Otro problema añadido al diseño de los moldes metálicos es la contaminación de cationes que se desprenden del ánodo durante el proceso, a expensas de los cuales se desarrolla sobre el electrodo de trabajo una capa de óxido que en algunos casos puede hacer variar las propiedades conductoras del metal, o que pueden incluso contaminar la muestra dándole color tras la cocción ( $\mathrm{Ni}, \mathrm{Fe}$, etc.). La pasivación de los metales que podrían ser usados como substrato en el proceso ha sido estudiada por Van der Poorten y col. [30-33] utilizando barbotinas de distintos materiales arcillosos. Una solución a este problema pasa por el diseño de moldes con base de grafito, que no estén sometidos a reacciones galvanostáticas. Pero el uso de este tipo de moldes puede producir el sobrecalentamiento del electrodo y su entorno por efecto Joule, provocando la aparición de un gradiente de humedad en la pieza, cuyas consecuencias son las mismas que se mencionaron en el párrafo anterior. Otra solución, adoptada en algunos prototipos industriales, es limpiar la capa de óxido formada sobre el substrato metálico cada vez que se repite el ciclo de deposición [34].

Sin embargo, la problemática que suscita el diseño de moldes se ve ampliamente recompensada con la versatilidad del método, ya que tanto a nivel de laboratorio como a nivel industrial, se han desarrollado equipos que permiten el conformado de piezas de formas complejas (ángulos rectos, esquinas, puntas, etc.), imposibles de conformar por colaje.

También la cinética del proceso supone un considerable ahorro, ya que como se ha comprobado con anterioridad, la ley que rige el crecimiento de espesor de la pieza en el conformado por electroforesis es lineal con el tiempo (fig. 7), mientras que en colaje varía linealmente con la raiz cuadrada del tiempo, y la cinética del proceso es mucho más rápida $[4,5,35]$, hasta el punto de obtener placas de $2 \mathrm{~m}^{2} \times 4 \mathrm{~mm}$ en sólo 3-4 minutos, y cilindros huecos de un espesor de $5 \mathrm{~mm}$ y una longitud de $8 \mathrm{~cm}$, en 5 minutos. La limitación de tiempo, y por tanto de espesor de las piezas, viene dada una vez más por el gradiente de humedad que provoca el continuo secado por electroósmosis del agua durante el proceso. Con todo ello, al final de la década de los 70 se desarrollaron ya los primeros prototipos de maquinaria industrial que permitían obtener tanto piezas longitudinales (losas, tejas, azulejos, etc.), como piezas volumétricas (sanitarios) [34, 36-37].

\subsection{Cerámica tradicional. Obtención de esmaltes por EPD en medio acuoso}

Sin duda, dentro del campo de la cerámica tradicional, el proceso en el que más se ha desarrollado la EPD en medio acuoso es en el esmaltado. Una de las ventajas más representativas que presenta este proceso es la posibilidad de automatización de la planta industrial, integrando los tres procesos fundamentales que completan el proceso de esmaltado (tratamiento de los substratos a recubrir, electrodeposición y aclarado) en un ciclo único de producción [38]. La automatización del proceso lleva consigo un considerable ahorro de tiempo, espacio, energía y materias primas, como demuestran varios estudios realizados sobre prototipos y plantas industriales implantadas en industrias como Ferro-ECA Electronics Co., 
DeDietrich Co, Eisenmann Corp o Bayer, avaladas por la creciente fabricación de este tipo de maquinaria por parte de empresas como Miele, Cannon, Esswein o DeEtna [39,40].

Un ciclo de producción de esmaltado por deposición electroforérica que incluya el pretratamiento del substrato tiene una duración aproximada de 8-10 min y ocupa $25 \mathrm{~m}^{2}$ en la planta de fabricación. Estos datos son significativos si se tiene en cuenta que tan sólo el equipo de pretratamiento por pulverización de los substratos ocupa $260 \mathrm{~m}^{2}$ y el ciclo completo de producción de la misma cantidad de material tiene una duración aproximada de una hora [8]. Al considerable ahorro que supone la reducción del espacio y el tiempo dedicado a la producción, se une el ahorro energético, ya que sólo se consumen $0,22 \mathrm{kWh}$ por $\mathrm{m}^{2}$ de área esmaltada, y la reducción de mano de obra, sin olvidar en ningún caso que el propio carácter del proceso favorece el reciclado de la materia prima sobrante que no ha sido depositada.

La integración de las distintas etapas del proceso se ha podido llevar a cabo gracias a las buenas propiedades de adhesión, densidad (humedad de la frita menor del 25\%), homogeneidad y resistencia mecánica y química de los depósitos obtenidos por EPD. Propiedades todas ellas indispensables, tanto para que el recubrimiento pueda ser sometido a los tratamientos posteriores a la deposición (aclarado, secado y cocción) como para obtener un buen acabado en el esmaltado de la pieza $[41,42]$. El esmaltado por deposición electroforética, frente a otros métodos de obtención de recubrimientos como inmersión o pulverización, ofrece la posibilidad de control sobre el crecimiento y uniformidad del espesor del recubrimiento, así como del diseño de la forma y de la superficie que se desea recubrir.

Por otra parte, la mayor desventaja que plantea el método, relativa fundamentalmente a la electrolisis del agua que tiene lugar de forma paralela a la deposición, puede evitarse realizando un estudio minucioso del sistema con el fin de establecer las condiciones de densidad de corriente adecuadas para favorecer el movimiento de las partículas sin sobrepasar el valor límite a partir del cual tiene lugar la electrolisis. Dicho valor puede ser determinado a partir de las curvas de polarización del electrodo. Para acondicionar el sistema se puede actuar sobre la defloculación de la barbotina (modificando su conductividad), sobre el tratamiento superficial del substrato o estableciendo un régimen de agitación sobre el sistema para favorecer el escape de burbujas producidas en la electrolisis, que de otra forma podrían perjudicar la homogeneidad del recubrimiento [43].

\subsection{Aplicaciones en cerámica avanzada}

Como se dijo anteriormente, la mayoría de los trabajos desarrollados en el campo de la cerámica avanzada se han llevado a cabo a partir de suspensiones en medios orgánicos $[1,18$ 21,44-46]. No obstante en algunos trabajos, el contenido en agua ha sido estudiado como un factor importante para la viabilidad del proceso en medio orgánico, por ejemplo, en la obtención de pantallas de fósforo $[47,48]$ o en la de piezas de alúmina [49-51]. Tomando este tipo de estudios como referencia y teniendo en cuenta las desventajas derivadas del uso del agua como vehículo de la suspensión, resulta sorprendente el creciente interés que ha despertado en la última década el conformado de materiales avanzados, incluso de estructuras complejas, por deposición electroforética a partir de suspensiones acuosas. Una muestra de los trabajos realizados y los materia-

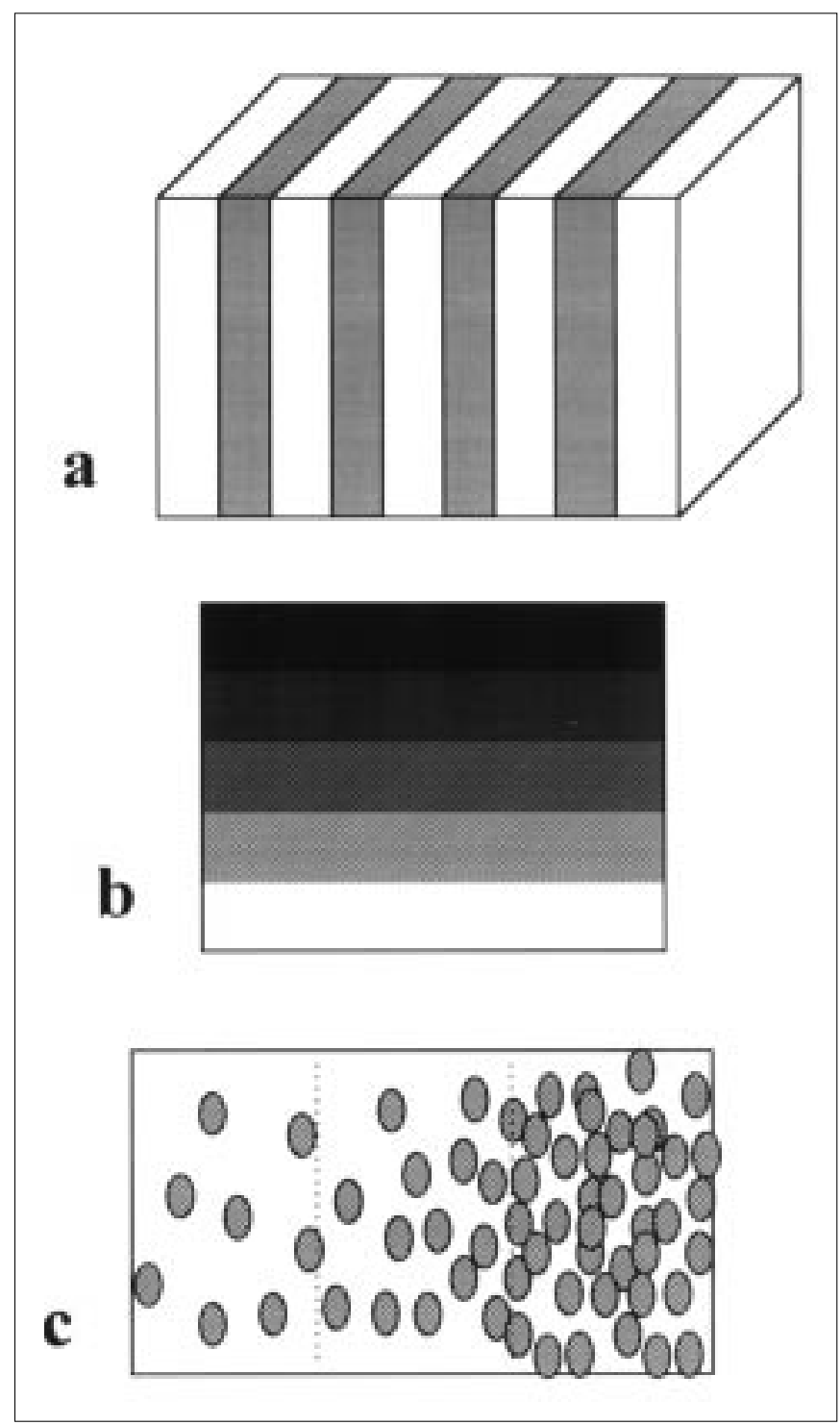

Fig. 13. Diseño de materiales compuestos cerámica-cerámica: laminados (a) y con función gradiente (b), y de materiales compuestos metal-cerámica con función gradiente (c).

les obtenidos se resumen en la tabla 1 .

Existen trabajos desarrollados en este campo que estudian la deposición de $\mathrm{Al}_{2} \mathrm{O}_{3}$ sobre substratos semiconductores de grafito, en los que se realiza un amplio estudio sobre las condiciones y cinética del proceso [9,10], y se evalúa la influencia de las condiciones de defloculación y de la capacidad de conducción eléctrica de la suspensión en las propiedades de los materiales obtenidos [52,24].

El uso de electrodos de trabajo semiconductores reduce algunas de las dificultades que plantea la EPD realizada en medio acuoso, sin embargo parece interesante la posibilidad que ofrece el método de cara al conformado de recubrimientos cerámicos sobre substratos metálicos. La posible obtención de este tipo de materiales pasa inevitablemente por el estudio de contaminación del depósito obtenido por difusión y migración de los cationes metálicos generados en el ánodo a lo largo del proceso [25].

Por otra parte, materiales que han suscitado un gran interés en la comunidad científica como los materiales compuestos con estructura laminada (fig. 13a), han sido conformados por EPD a partir de suspensiones orgánicas por Nicholson y col. 
$[46,53,54]$, mientras que otros grupos de investigación han demostrado que existe la posibilidad de obtener este tipo de materiales microlaminados a partir de suspensiones acuosas [55,56], sin perder la homogeneidad y características estructurales de las multicapas obtenidas en los trabajos realizados en medio orgánico.

Las cerámicas no oxídicas, caracterizadas por su dificultoso procesamiento, también han sido conformadas por EPD. Hasta el momento se han realizado estudios sobre la capacidad de deposición electroforética de $\mathrm{SiC}[11,57,58]$ como reforzamiento contra la oxidación y erosión de fibras de C. La deposición de distintas cantidades de $\mathrm{SiC}$ sobre fibras de grafito, combinada con un proceso de sinterización con presión de los distintos materiales obtenidos, dan lugar a la obtención de materiales con función gradiente en su contenido en C/SiC (fig. 13b).

Otros tipos de materiales compuestos como las estructuras de matríz metálica y fase dispersa cerámica, en los que el cerámico actúa como reforzamiento contra el desgaste y la abrasión, han sido obtenidos por codeposición electroforética en agua. En el procesamiento de este tipo de materiales compuestos se aprovecha la posibilidad que ofrecen tanto los metales como las cerámicas, de ser conformados por EPD al aplicar un campo eléctrico a una suspensión de partículas cerámicas dispersas en soluciones acuosas de cationes metálicos, provocando así la codeposición de ambos materiales. En el trabajo realizado por Merk y col. se describe la obtención de recubrimientos de matriz metálica $(\mathrm{Ni}, \mathrm{Cu})$ reforzados con una fase dispersa de $\mathrm{SiC}$ [59] o $\mathrm{Al}_{2} \mathrm{O}_{3}$ [60] para mejorar su comportamiento a la abrasión. Siguiendo esta interesante línea de investigación, se ha aprovechado la filosofía de los materiales con función gradiente para paliar el posible deterioro de las condiciones de adherencia de este tipo de recubrimientos. Para ello, se juega con el contenido en volumen de material cerámico disperso en la matriz, que aumenta a medida que nos alejamos del substrato (fig 13c), para evitar el efecto de falta de adherencia que provoca la diferencia entre los coeficientes de dilatación del substrato (metal) y del depósito (cerámicametal). Este efecto se consigue al procesar el material por electrodeposición, simplemente aplicando un gradiente creciente de intensidades sobre la suspensión. De esta forma, la deposición de uno $\mathrm{u}$ otro materiales queda favorecida debido a las diferentes cinéticas de deposición que presentan ambos.

La versatilidad en cuanto al tamaño y forma del substrato a recubrir se aprovecha en campos como la electrocerámica y la biocerámica. La obtención de recubrimientos porosos de fosfato de calcio bioactivo que faciliten la interacción con el entorno biológico para su utilización en prótesis ortopédicas, es otra de las más novedosas aplicaciones de esta técnica. En este campo se ha hecho hincapié en la posibilidad de obtener estos difíciles recubrimientos sobre substratos porosos de aleaciones

TABLA I.

MATERIALES OBTENIDOS POR DEPOSICIÓN ELECTROFORÉTICA EN MEDIO ACUOSO.

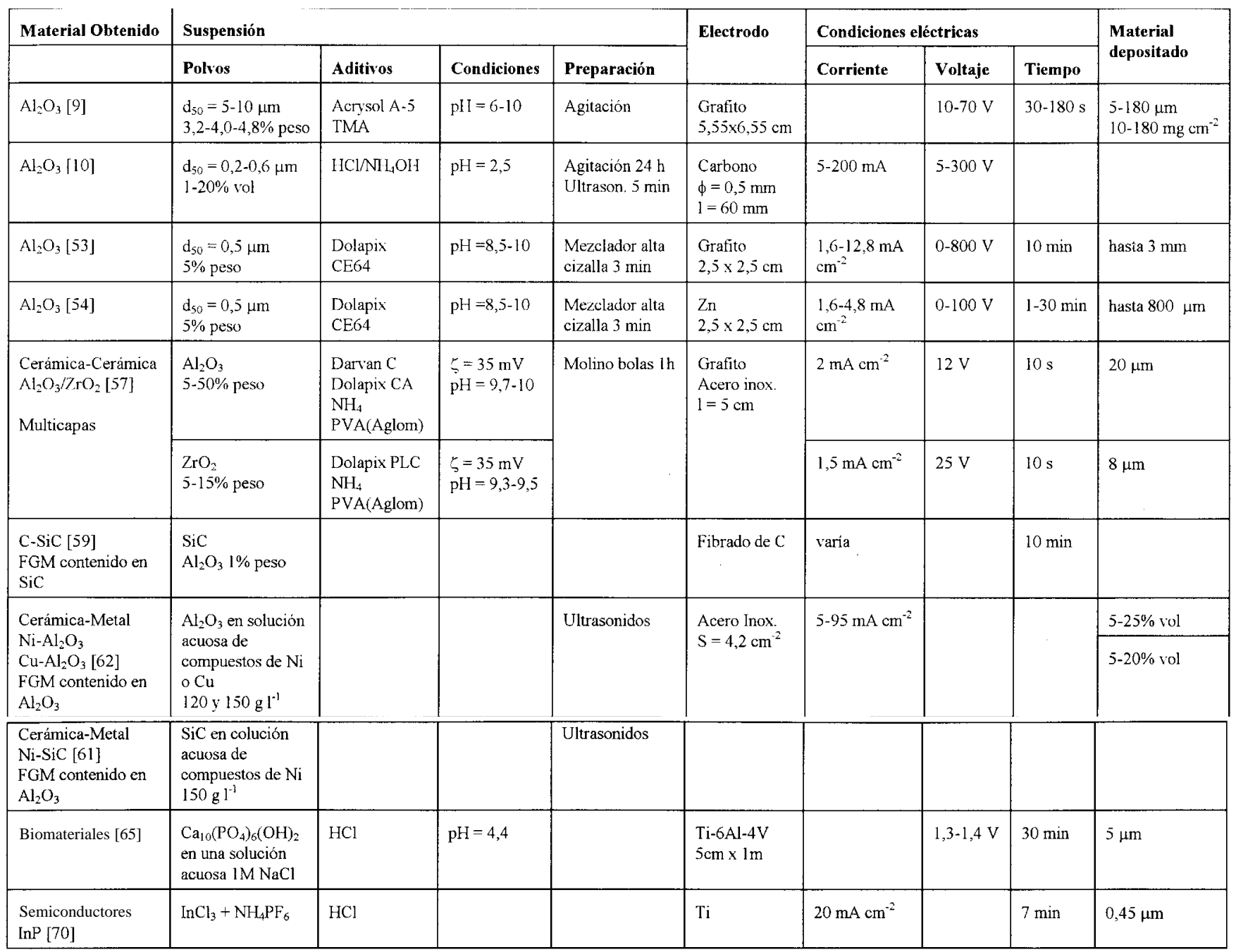


Ti-6Al-4V $(5 \mathrm{~cm} \times 1 \mathrm{~cm})$, principalmente a partir de medios orgánicos [61, 62], o bien a partir de disoluciones de polvo de fosfato de calcio tribásico $\left(\mathrm{Ca}_{10}\left(\mathrm{PO}_{4}\right)_{6}(\mathrm{OH})_{2}\right)$ en una disolución acuosa de $\mathrm{NaCl} 1 \mathrm{M}$ [63].

En la misma línea, entre los materiales electrocerámicos procesados por EPD más conocidos se encuentran los supercondutores del sistema $\mathrm{YBaCuO}$, aunque también se procesan por esta técnica materiales como el Titanato de Bario, PZT, semiconductores... [64-69], algunos de los cuales se han obtenido también a partir de suspensiones en medios acuosos [68].

Por utimo, cabe destacar que gran parte de los materiales funcionales desarrollados en el campo de la electrocerámica se obtienen por deposición electrolítica a partir de disoluciones haciendo reaccionar determinados compuestos en disolución en el entorno del electrodo. El caso más representativo es el procesamiento de superconductores, en los que en ocasiones, en lugar de partir de suspensiones coloidales del polvo previamente sintetizado, se preparan disoluciones de los distintos cationes del sistema manteniendo la estequiometría del compuesto que se pretende depositar. Tanto las suspensiones como las disoluciones de estos materiales se preparan por lo general en medio orgánico para no dañar las propiedades intrínsecas del material final. No obstante, ya fuera del campo de los supercondutores, Gal-Or y col. [70- 74] han abierto una prometedora línea de investigación sobre la deposición de $\mathrm{Al}_{2} \mathrm{O}_{3}, \mathrm{ZrO}_{2}, \mathrm{ZrTiO}_{4}$ a partir de las disoluciones de los cationes correspondientes.

\section{SUMARIO}

En este trabajo se ha pretendido poner de relieve las características más destacadas de una técnica de conformado que, aunque conocida desde hace tiempo en la indutria de la cerámica tradicional, ha despertado un enorme interés en la manufactura de materiales cerámicos de microestructura compleja (como laminados o materiales con función gradiente) y de materiales compuestos de matriz cerámica, metálica e incluso polimérica.

Ha de destacase el hecho de que la técnica de EPD en agua es ya considerada convencional en el campo de la cerámica tradicional, mientras que en el caso de la cerámica avanzada está comenzando a dar sus primeros frutos. En este campo la mayor parte de los trabajos ( $>90 \%$ ) se centran en el estudio de sistemas no acuosos, con los problemas asociados a los mismos, relativos al elevado costo, toxicidad y efectos medioambientales, por lo que el uso de sistemas acuosos se perfila como una técnica de procesamiento clave para abordar de forma competitiva las crecientes demandas de las futuras tecnologías.

\section{AGRADECIMIENTOS}

Este trabajo ha sido financiado por la CICYT, proyecto MAT97-0676.

\section{BIBLIOGRAFÍA}

1. H. C. Hamaker, "Formation of a deposit by electrophoresis", Trans. Faraday Soc., 36 279-87 (1940).

2. H. Koelmans y J. Th. G. Overbeek, "Stability and electrophoretic deposition of suspensions in non-aqueuos media", Discuss Faraday Soc., 18 52-63 (1954).

3. J. J. Shyne y H. G. Scheible, "Electrophoretic coatings", pp. 714-30, in Modern Electroplating, 2nd edition, Edited by J. Wiley \& Sons, New York, 1963.

4. J. C. Descamps, H. Vander Poorten, A. Depellegrin, C. Cazzro, V. Vandeneede y X. Y. Xiong, "Preparation, structure et propiétès mécaniques d'une ceramique élèctrophormée", Silicates Ind., 55 41-54 (1990).

5. W. Ryan y E. Massoud, "Electrophoretic deposition could speed up ceramic casting", Interceram, 28 (2) 117-19 (1979).

6. H. Vander Poorten y G. Moortgat-Hasthorpe, “Aptitude a l'electroformage de diverses argiles de la Communaute Europeenne, comparaison avec le coulage en moule de platre", Silicates Ind., 53 109-15 (1988).

7. M. Boncoeur y S. Carpentier, "Le formage par depot electrophoretique", L'industrie Ceramique, 648 79-81 (1972).

8. H. Warnke y N. F. A. Hoens, "New perspectives in the electrophoretic enameling process by tailormade pretreatment and technical improvements", Ceram. Eng. Sci. Proc., 6 335-51 (1985).

9. J. Y. Choudhary, H. S. Ray y K. N. Rai, “Electrophoretic depositon of Alumina from aqueous suspensions", Trans. J. Br. Ceram. Soc., 81 193-96 (1982).

10. Y. Hirata, A. Nishimoto y Y. Ishihara, "Forming of Alumina powder by electrophoretic deposition", J. Ceram. Soc. Jpn., Int. Ed., 99 105-09 (1991)

11. C. Kawai, S, Wakamatsu, S. Sakagami y T. Nomura, "Fabrication of continuos fibrer inforced $\mathrm{SiC}$ matrix composites with gradient compositions", J. Mater. Sci. Lett., 12 1156-59 (1993).

12. R. Moreno,The role of slip additives in tape-casting technology: solvents and dispersants. Am. Ceram. Soc. Bull., 71 (10) (1992) 1521-31.

13. S. Baudet, “Les mécanismes de la défloculation (suite)”, L'Industrie Ceramique, 754 (10) 712-23 (1981).

14. R. Moreno, J.S. Moya y J. Requena, “Electroquímica de suspensiones cerámicas", Bol. Soc. Esp. Ceram. Vidr., 26 (6) 355-65 (1987).

15. P. S. Nicholson y P. Sarkar, "The electrophoretic deposition of ceramics", in Ceramic TransactionProccedings of the Conference on Ceramic Processing Science and Technology, Friedriscshafen, Germany, 11-14 Sept., 1994.

16. P. Sarkar y P. S. Nicholson, "Electrophoretic deposition (EPD): mechanisms, kinetics, and application to ceramics", J. Am. Ceram. Soc., 79 (8) 1897-2002 (1996).

17. W.D. Kingery, H.K. Bowen y D.R. Uhlmann, "Introuction to Ceramics, Second Edition", Ed. John Wiley \& Sons (1976) p.848.

18. A. I. Avgustinik, V. S. Vigdergauz y G. I. Zhuravlev, “Electrophoretic deposition of ceramic masses from suspensions and calculation of deposit yields", J. Appl. Chem. (URSS), 35 2090-93 (1962).

19. D. U. Krishna Rao y E. C. Subbarao, "Electrophoretic deposition of Magnesia", Am. Ceram. Soc. Bull., 58 (4) 467-69 (1979).

20. J. Mizuguchi, "Dependence of the diffusion current on the degree of polymerization of Nitrocellulose in Acetone-Isopropyl Alcohol", J. Electrochem. Soc., 129 1520-23 (1982).

21. J. H. Jean, "Electrophoretic deposition of $\mathrm{Al}_{2} \mathrm{O}_{3}-\mathrm{SiC}$ composites", Mater. Chem. Phys., 40 285-90 (1995).

22. B. Ferrari y R Moreno, “EPD of Y-TZP powders from aqueous media on $\mathrm{Ni}$ sustrates", en redacción.

23. B. Ferrari, R, Moreno y P. S. Nicholson, “Electrophoretical Deposition of MgO powders", en redacción.

24. B. Ferrari y R. Moreno, "Electrophoretic deposition of alumina slips", J. Eur.Ceram. Soc., 17 549-56 (1997).

25. B. Ferrari, R. Moreno y, J.F. Fariñas, "Influencia del procesamiento en la con-

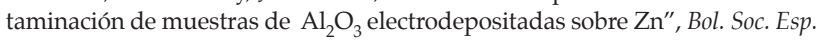
Ceram. Vidr., 36 (1) 31-37 (1997).

26. B. Ferrari. "Conformado de materiales cerámicos por deposición electroforética", Tesis Doctoral Universidad Politécnica, ETSI Minas, Madrid 1998.

27. X. Y. Xiong y H. Vander Poorten, "Electroformage en ceramique sous pression", Silicates Ind., 11-12 327-31 (1994).

28. W. Ryan, E. Massoud y C. T. S. B. Perera, "Fabrication by electrophoresis", pp 46-47, Symposium of the Casting Process, 1980.

29. M. Mihailescu, M. Emadi, V. Vancea y M. Marcu, “Electrophoretic behavior of ceramic sanitaryware slips", Interceram, 40 165-70 (1991).

30. H. Vander Poorten, G. Moortgat-Hasthorpe y M. Mercier, “Electrochimie des barbotines", Silicates Ind., 40 (4-5) 89-96 (1980).

31. H.Vander Poorten, “Caracterisation del'electrodeposition et des electrodepots de pates ceramiques", Silicates Ind., 41 (9) 159-72 (1981)

32. H. Vander Poorten y G. Moortgat-Hasthorpe, "Propietes electrochimiques de diverses argiles de la Communaute Europeenne, etude plus particuliere des phenomenes catodiques", Silicates Ind., 52 13-19 (1987).

33. H. Vander Poorten y G. Moortgat, "Propietes electrochimiques de diverses argiles de la Communaute Europeenne, etude plus particuliere des phenomenes anodiques", Silicates Ind., 52 155-69 (1987).

34. M. S. Chonberg y F. Handie, "Processes and equipment for the production of materials by electrophoresis Elephant", Interceram, 133-34 (1978)

35. M. Aveline, "Façonnage par électrophorese", L'Industrie Céramique, 58128 31 (1966).

36. M. S. Chonberg, "Possibilites de l'electrophorese pour faconnage des produits ceramiques", L'industrie Ceramique, 718 (6) 423-26 (1978).

37. F. Händle, "Electrophoretic forming of ceramic materials", Keram.Z., 32 (4) 185-88 (1980). 
38. A. Betz y K. O. Helm, "Electroporcelain enameling", Ceram. Eng. Sci. Proc., 9 380-85 (1988).

39. J. A. Olenick y W. Joseph, "Electrophoretic enameling - an area of steady advancement", Ceram. Eng. Sci. Proc., 3 284-90 (1982).

40. E. W. Hughes y D. F. Thomas, "Electodeposition in the United States", Ceram. Eng. Sci. Proc., 9 391-93 (1988)

41. J.P. Passaquet y J.L. Saulle, "Emaillage par electrophorèse", L'Industrie Céramique, 698 595-97 (1976)

42. F. C. Sas y D. Greewood, "Electrphoresis update”, Ceram.Eng. Sci. Proc., 14 (56) $116-18$ (1993).

43. H. Hoffmann. "Theory and practice of electrocoating of porcelain enamel", Am. Ceram. Soc. Bull., 57 605-08 (1978).

44. L. Vandeperre, O. Van Biest y W.J. Clegg, "Silicon Carbide laminates with carbon interlayers by electrophoretic deposition", Key Engineering Materials, Vols. 127-131, pp. 567-74, Ed. Trans Tech Publications, Switzerland, 1997.

45. A. R. Boccaccini, D. N. Boccaccini y C. B. Ponton, “Fabricación de materiales cerámicos compuestos con refuerzos de tejidos de fibras de carburo de silicio usando deposición electroforética", Bol. Soc. Esp. Ceram. Vidr., 35 (4) 257-63 (1996).

46. P. Sarkar, O. Prakash, G. Wang y P. S. Nicholson, "Microlaminate ceramic/ceramic composites ( $\mathrm{YSZ} / \mathrm{Al}_{2} \mathrm{O}_{3}$ ) by electrophoretic deposition", Ceram.Eng. Sci. Proc., 15 (5) 1019-27 (1994).

47. M. J. Shane, J. B. Talbot, R. D. Schreiber, C. L. Ross, E. Sluzky y K. R. Hesse, "Electrophoretic deposition of Phosphors", J. Colloid Interfase Sci, 165 325-33 (1994).

48. M. J. Shane, J. B. Talbot, R. D. Schreiber, C. L. Ross, E. Sluzky y K. R. Hesse, "Electrophoretic deposition of Phosphors", J. Colloid Interfase Sci, 165 334-40 (1994)

49. J. H. Kennedy y A. Foissy, "Measurement of mobility and zeta potential of beta-Alumina suspensions in various solvents", J. Electrochem. Soc., 60 33-36 (1977).

50. R. W. Powers, S. P. Mitoff, R. N. King y J. C. Bielawski, “A beta-Alumina fabrication scheme with some special features", Solid State Ionics, 5 287-90 (1981).

51. F. Harbach, R. Neeff, H. Neinburg y L. Weiler, "Reliable ceramic components from colloidal suspensions", cfi/Ber. DKG, 67, 130-35 (1990).

52. B. Ferrari y R. Moreno, "The conductivity of aqueous $\mathrm{Al}_{2} \mathrm{O}_{3}$ slips for electrophoretic deposition", Mater. Lett., 28 (4-6) 353-55 (1996).

53. P. S. Nicholson, P. Sarkar y S. Datta, "Producing ceramic laminate composites by EPD", Am. Ceram. Soc. Bull., 75 (11) 48-51 (1996).

54. P. Sarkar, X. Haung y P. S. Nicholson, "Zirconia/Alumina functionally gradiented composites by electrophoretic deposition techniques", J. Am. Ceram. Soc., 76 (4) 1055-56 (1993)

55. R. Fisher, E. Fisher, G. De Portu y E. Roncari, "Preparation of ceramic microlaminate by electrophoresis in aqueous system", J. Mater. Sci. Lett., 14 25-27 (1995).

56. B. Ferrari, A.J. Sanchez-Herencia y R. Moreno, “Aqueous electrophoretic deposition of $\mathrm{Al}_{2} \mathrm{O}_{3} / \mathrm{ZrO}_{2}$ layered ceramics", Mat. Res. Bull., 33 487-99 (1998).

57. C. Kawai y S. Wakamatsu, "Synthesis of a functionally gradient material based on C/C composites using an electrodeposition method", J. Mater. Sci. Lett., 14 467-69 (1995).

58. C. Kawai y S. Wakamatsu, "Fabrication of $\mathrm{C} / \mathrm{SiC}$ composites by an electrodeposition/sintering method and control of the properties", J. Mater. Sci., 31 2165-70 (1996)
59. N. Merk, "Electron microscopy study of the thermal decomposition in Ni-SiC electrodeposits", J. Mater. Sci. Lett., 14 592-95 (1995).

60. X.M. Ding, N. Merk y B. Ilschner, "Particle volume graded $\mathrm{Ni}-\mathrm{Al}_{2} \mathrm{O}_{3}$ and $\mathrm{Cu}-$ $\mathrm{Al}_{2} \mathrm{O}_{3}$ composite deposits: production and performances", Actas del $3 \mathrm{rd}$ International Symposium on structural and Functionally Gradient Materials (FGM3), pp 365-70, Vol. 1, ed. B. Ilchner, N. Cherradi Laussanne, Switzerland, 1994.

61. P. Ducheyne, M. Van Raemdonck, J. C. Heughebaert y M. Heughebaert, "Structural analysis of hydroxyapatite coatings on Titanium", Biomaterials, 7 97-103 (1986).

62. P. Ducheyne, S. Radin, M. Heughebaert y J. C. Heughebaert, “Calcium Phosphate ceramic coatings on porous Titanium: effect of structure and composition on electrophoretic deposition, vacumm sintering and in vitro dissolution", Biomaterials, 11 244-54 (1990).

63. M. Shirkhanzadeh, "Bioactive Calcium Phosphate coatings prepared by electrodeposition", J. Mater. Sci. Lett., 10 (23) 1415-17 (1991).

64. J. Mizuguchi, M. Suzuki, H. Yamato y M. Matsumura, "Characterization of $\mathrm{YBa}_{2} \mathrm{Cu}_{3} \mathrm{O}_{7-\mathrm{x}}$ layers prepared by electrophoretic deposition", J. Electrochem. Soc., 138 2942-46 (1991).

65. D.N. Bhattacharya, S. N. Roy, R. N. Basu, A. Das Sharma y H. S. Maiti, "Critical current in electrophoretically deposited thick films of YBCO superconductor", Mater. Lett., 16 337-41 (1993).

66. D. N. Bhattacharya y D. S. Ginley, "Preparation of Lead-Zirconium-Titanium film and powder by electrodeposition", J. Electrochem. Soc., 143 (1) 97-99 (1996).

67. M. Nagai, K. Yamashita, T. Umegaki y Y. Takuma, “Electrophoretic deposition of Ferroelectric Barium Titanate thick films and their dielectric properties", J. Am. Ceram. Soc., 76 (1) 253-55 (1993).

68. N. Sahu, "Aqueous electrodeposition of InP semiconductor films", J. Mater. Sci. Lett., 8 (5) 533-34 (1989).

69. F. Linder y A. Feltz, Athin layer NTC semiconductor ceramics based on $\mathrm{NiMn}_{2} \mathrm{O}_{8}$ and $\mathrm{Zn}_{\mathrm{z}} \mathrm{NiMn}_{2-\mathrm{z}} \mathrm{O}_{4}(\mathrm{z}=1 / 3,2 / 3)^{\prime \prime}$, J. Eur. Ceram. Soc., 11 269-74 (1993).

70. L. Gal-Or, I.Silberman y R. Chaim, "Electrophoretic $\mathrm{ZrO}_{2}$ coatings", J. Electrochem. Soc., 138 1939-46 (1991)

71. S. Haber y L. Gal-Or, "Deep electrophoretic penetration and deposition of ceramic particles inside porous substrates. I. Analytical model", J. Electrochem. Soc., 139 1071-77 (1992).

72. L. Gal-Or, S. Liubovich y S. Haber, "Deep electrophoretic penetration and deposition of ceramic particles inside porous substrates. II. Experimental model", J. Electrochem. Soc., 139 1078-81 (1992).

73. R. Chaim, G. Stark y L. Gal-Or, "Electrochemical $\mathrm{ZrO}_{2}$ and $\mathrm{Al}_{2} \mathrm{O}_{3}$ coatings on $\mathrm{SiC}$ substrates", J. Mater. Sci., 29 6241-48 (1994).

74. I. Zhitomirsky, L. Gal-Or y S. Klein, "Electrolytic deposition of $\mathrm{ZrTiO}_{4}$ films", J. Mater. Sci. Lett., 14 60-62 (1995).

Recibido: 15-10-96

Aceptado: 1-4-98

\section{ECASIA 99}

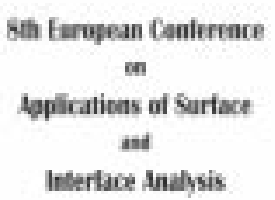

first Nanowacereft

\section{E C A S I A' 99}

Sevilla, Spain, 4-8 October 1999

Para más información:

ECASIA'99

Departamento Física Aplicada, C-XII

Facultad de Ciencias. Universidad Autónoma de Madrid

Cantoblanco E28049 (Madrid) Spain

Fax +34-1-3973969

E- mail: josem.sanz@uam.es 